\title{
Temperature Stratification in a Cryogenic Fuel Tank
}

\author{
Matthew J. Daigle ${ }^{1}$ and Vadim N. Smelyanskiy ${ }^{2}$ \\ NASA Ames Research Center, Moffett Field, CA, 94035, USA \\ Jacob Boschee ${ }^{3}$ and Michael Foygel ${ }^{4}$ \\ South Dakota School of Mines and Technology, Rapid City, SD, 57701, USA
}

A reduced dynamical model describing temperature stratification effects driven by natural convection in a liquid hydrogen cryogenic fuel tank has been developed. It accounts for cryogenic propellant loading, storage, and unloading in the conditions of normal, increased, and micro- gravity. The model involves multiple horizontal control volumes in both liquid and ullage spaces. Temperature and velocity boundary layers at the tank walls are taken into account by using correlation relations. Heat exchange involving the tank wall is considered by means of the lumped-parameter method. By employing basic conservation laws, the model takes into consideration the major multi-phase mass and energy exchange processes involved, such as condensation-evaporation of the hydrogen, as well as flows of hydrogen liquid and vapor in the presence of pressurizing helium gas. The model involves a liquid hydrogen feed line and a tank ullage vent valve for pressure control. The temperature stratification effects are investigated, including in the presence of vent valve oscillations. A simulation of temperature stratification effects in a generic cryogenic tank has been implemented in Matlab and results are presented for various tank conditions.

\footnotetext{
${ }^{1}$ Research Computer Scientist, Intelligent Systems Division, NASA Ames Research Center, Moffett Field, CA 94035, USA.

${ }^{2}$ Senior Research Scientist, Applied Physics Group Lead, NASA Ames Research Center, Moffett Field, CA 94035, USA.

${ }^{3}$ Graduate Student, Physics Department, South Dakota School of Mines and Technology, Rapid City, SD, 57701, USA.

${ }^{4}$ Professor, Physics Department, South Dakota School of Mines and Technology, Rapid City, SD, 57701, USA.
} 


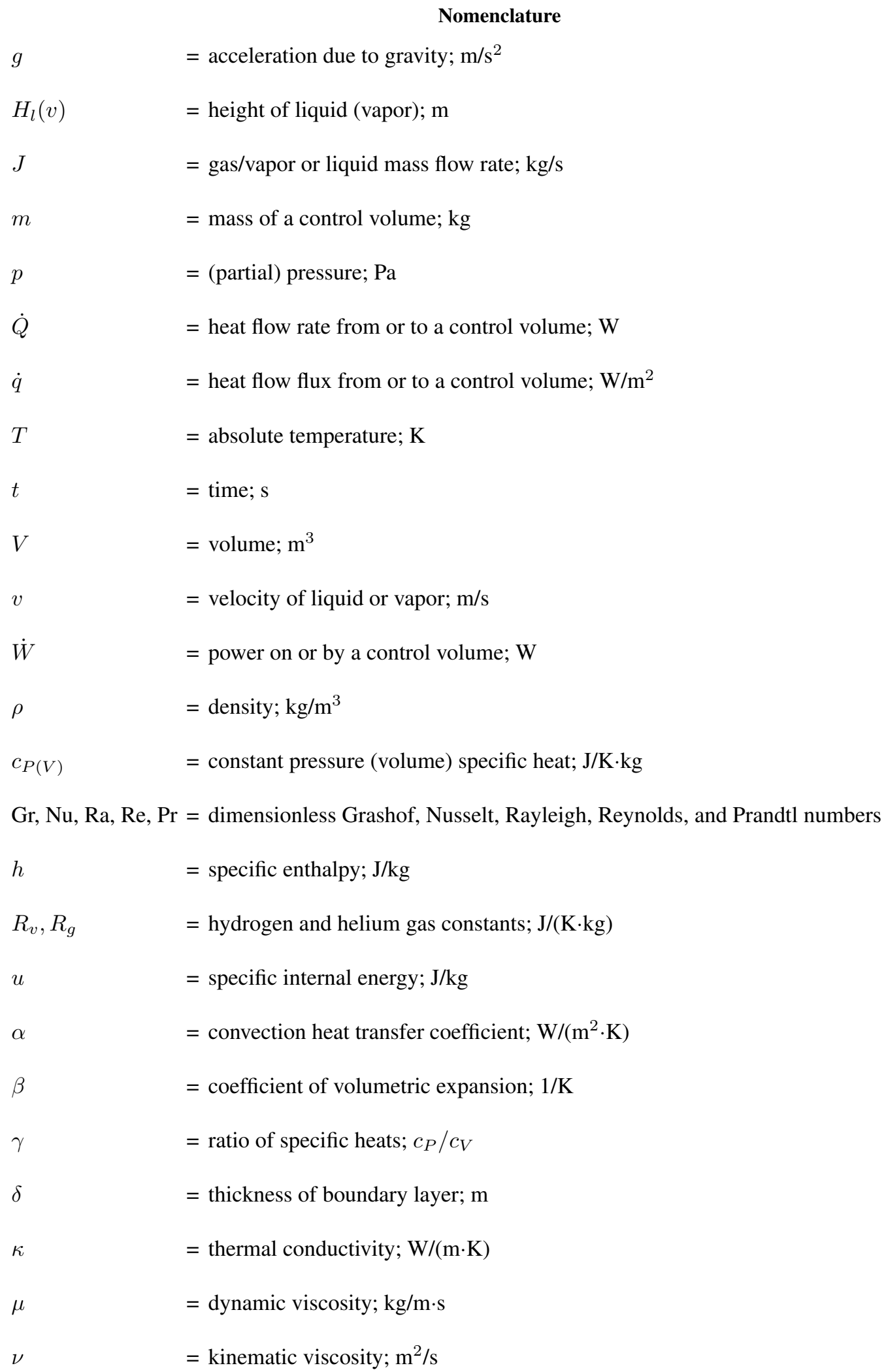




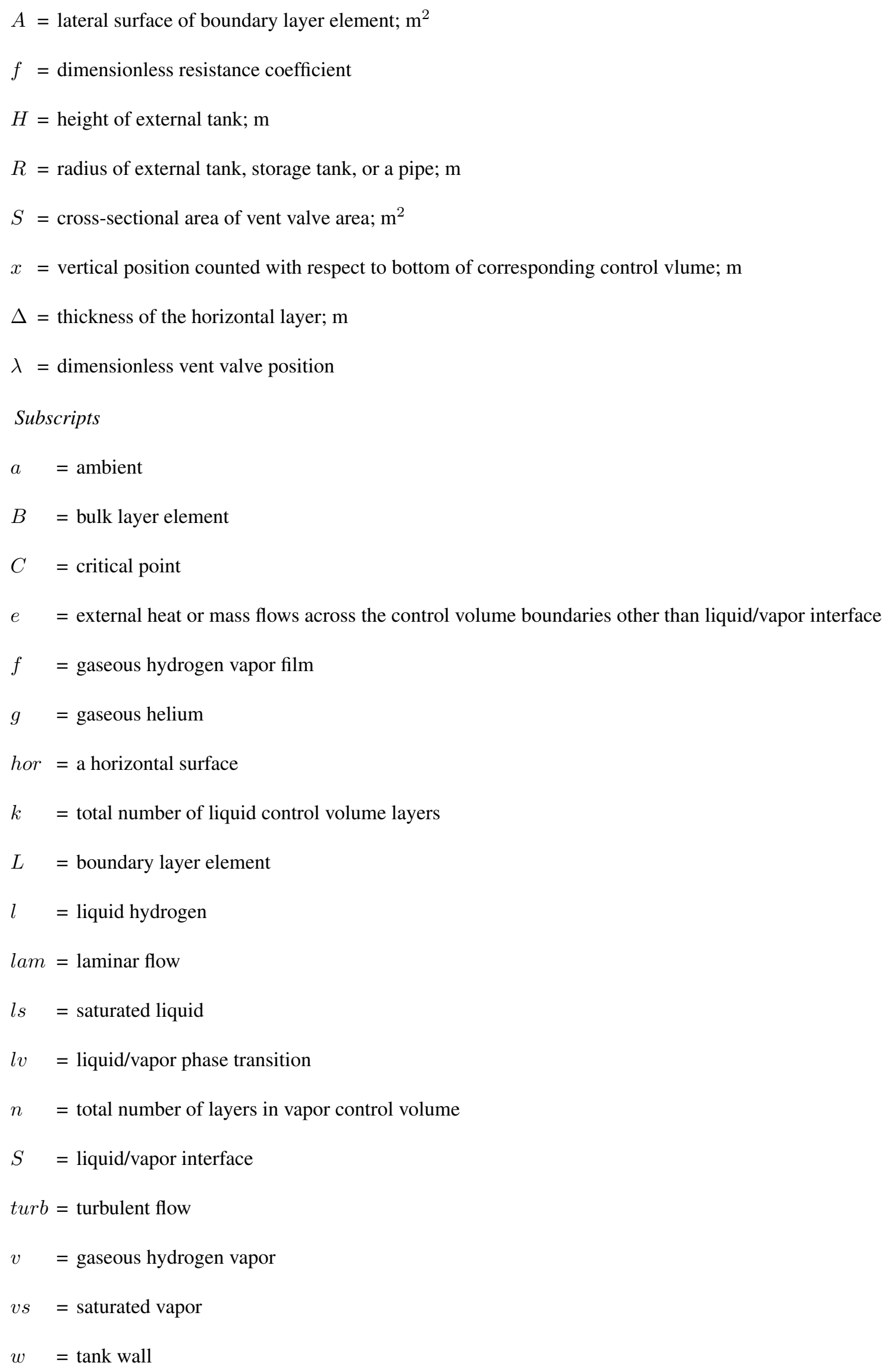




\section{Superscripts}

cond $=$ conduction

$\operatorname{conv}=$ convection

$\lambda \quad=$ gaseous hydrogen or helium

\section{Introduction}

Recently, a dynamical model was developed for a complex spatially-distributed system of liquid hydrogen (LH2) loading that involves the storage (ST) and external (ET) tanks as well as the transfer line [1]. This reduced model is based on a set of coupled ordinary integro-differential equations, which is shown to be well-suited to describing a generic cryogenic loading system, including one used for Space Shuttle fueling in both the nominal and major faulty regimes. The model accounts for the pressurizing helium gas injected into the ET and can be easily modified for similar cryogenic propellant loading systems.

Analysis has been performed for both the nominal regime and the effects of several primary faults, such as gas leaks in the ullage space of both the ST and ET, as well as clogging of the ET vent valve on the history of both tanks $[1,2]$. It is found that each of the faults is characterized by quite a pronounced dynamics. The most interesting observation is that some of the faults, such as a substantial clogging of the ET vent valve, usually have a slight effect on the integral variables, namely on the volumes of LH2 in the tanks or even on the ullage temperatures and pressures. Yet, the dynamics of the ET vent valve switching that causes pressure oscillations are shown to be extremely sensitive even to small deviations from the nominal regime and can be used for early fault identification by means of real time sensor data analysis. (The valve in question works between two threshold positions in order to maintain a required ullage pressure [1].) It means that one can not only detect, isolate and identify different faults based on the analysis of the sensor data characterizing the filling dynamics, but also infer the parameters of the model to be further used for prediction of future behavior of the system [2].

However, the above model, allowing for a fairly adequate description of the ET ullage history [1, 2], does not account for a substantial temperature stratification that may happen [3-6] in both the ullage [7-10] and the liquid $[9,10]$ control volumes of the external and storage cryogenic tanks. The major goal of the present paper is to develop a model that correctly describes possible thermal stratification in both the ullage and liquid bulk 
parts of a tank that is based on ordinary differential equations, similar to those discussed in [1], short of a fullscale computational fluid dynamics (CFD) modeling scheme [3-8], so that it can be implemented efficiently and used for online state estimation, fault diagnosis, and fault prognosis. A distinctive feature of this model is that it takes into consideration non-equilibrium mass and energy exchange processes of condensationevaporation at the liquid-vapor interface between the liquid and gas, and the thermal convection in both the liquid and vapor parts of the tank, which is driven by complex phenomena developing in the boundary layers adjacent to the tank walls as well as to the liquid-vapor interface.

The temperature stratification effects under consideration may be responsible for the possible appearance of the overheated layers $[11,12]$ of cryogenic fuel in the external tanks, and would be crucial to designing effective pressure and temperature control in the storage tanks [7-10,13]. The model is meant to be applied towards treating very important aspects of cryogenic fuel management technology [13], such as the minimization of the boil-off effects and the optimization of fuel bias, i.e. of the excess of fuel load above nominal aimed at preventing the cryogenic fuel from overheating due to radiation from the hot jet and heat conduction transfer through the ET walls from the engine, thrust cone, and common bulkhead. The overheated cryogenic liquid on its way to the engine may reach a pump impeller thus leading to the development of violent cavitation [14] in the areas where pressure is less than the saturated pressure of the overheated fuel.

The contributions of this work are as follows. We develop a general model of a cryogenic tank including temperature stratification effects in the liquid, vapor, and tank control volumes, for an arbitrary number of lumped-parameter layers. We develop a simulation that implements the model and allows the investigation of the dynamical behavior under both storage, loading, and unloading regimes. In particular, we show that the presence of a vent valve in the tank used for the purposes of maintaining pressure creates temperature waves that propagate through the layers. Simulation results are presented and detailed analysis is provided.

The paper is organized as follows. Section II describes the cryogenic tank model, which incorporates temperature stratification effects driven by convection in both the liquid and vapor parts of the tank. Section III deals with major results of our simulations of the LH2 cryogenic tank in different performance regimes. Section IV concludes the paper. 


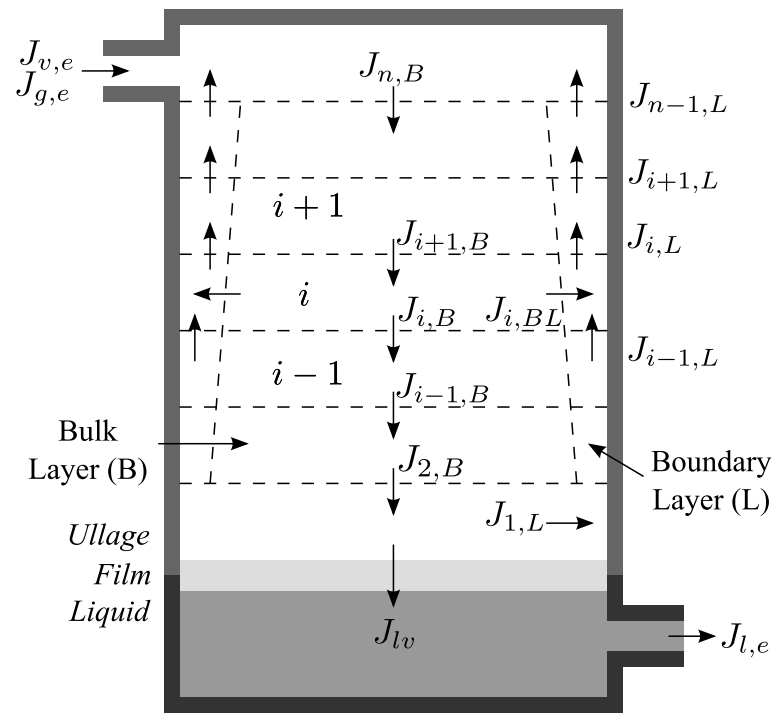

(a) Side view.

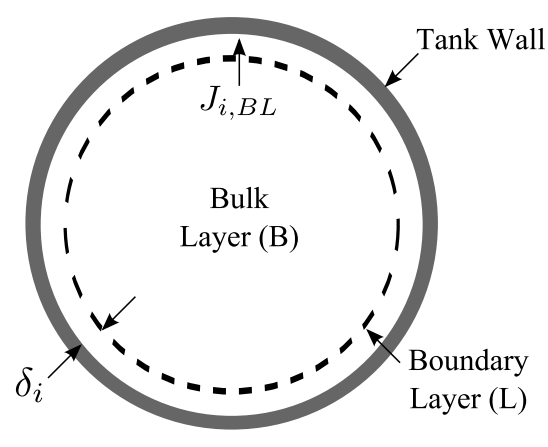

(b) Top view.

Fig. 1 Mass flow in a propellant tank with three control volumes (vapor, film, and liquid).

\section{Description of the Model}

Consider a cylindrical tank of radius $R$ where the gravity/inertia force is parallel to its axis. It is partially filled with liquid hydrogen (LH2); its ullage volume is filled by a mixture of gaseous hydrogen (GH2) and gaseous helium (GHe). The liquid and ullage (vapor) control volumes (CVs) are separated by a thin massless film of saturated hydrogen [1] (see Fig. 1). The following subsections describe the liquid and vapor CVs and the heat and mass flows depicted in Fig. 1.

\section{A. Vapor Control Volume}

Divide the vapor (ullage) CV into $n \geq 3$ layers perpendicular to the axis of the tank. The lowest one $(i=1)$ is adjacent to the saturated film CV and exchanges mass and energy with it. The upper one $(i=n)$ is at the top of the tank and exchanges heat with the top wall of the tank, as well as mass and energy with the environment through the vent valve, as shown in Fig. 1 . Any internal horizontal slice $(i=2, \ldots, n-1)$, in turn, consists of two sub volumes: (i) a torus-shaped element of the boundary layer $(L)$, which is adjacent to the lateral wall of the tank and exchanges heat with it; and (ii) the cylinder-shaped bulk element $(B)$, which is coaxial with the tank vertical axis $x$. Thus the total number of the vapor CVs is equal to $2(n-2)+2=$ $2(n-1)$.

In the presence of the two gas components in the vapor CV: GH2 $(v)$ and GHE $(g)$, for the $i$ th internal 
vapor bulk ( $B$ ) element (see Fig. 1), the mass and the energy conservation, respectively, yield [15-21]

$$
\begin{aligned}
\dot{m}_{i, B}^{(\lambda)} & =J_{i+1, B}^{(\lambda)}-J_{i, B}^{(\lambda)}-J_{i, B L}^{(\lambda)} \\
\frac{d}{d t} \sum_{\lambda=v, g} m_{i, B}^{(\lambda)} u_{i, B}^{(\lambda)} & =-\dot{W}_{i, B}^{(v)}+\sum_{\lambda=v, g} J_{i+1, B}^{(\lambda)} h_{i+1, B}^{(\lambda)}-\left(J_{i, B}^{(\lambda)}+J_{i, B L}^{(\lambda)}\right) h_{i, B}^{(\lambda)}
\end{aligned}
$$

where $\lambda=v, g$. Here, when the tank walls are hotter than the gas/vapor $\left.\left(T_{w v}>T^{(} v\right)_{i, B}\right)$, the flow vertically enters the element $(i, B)$ from the above layer $(i+1, B)$ and exits to an underlying bulk element $(i-1, B)$; the remaining part $(i, B L)$ spreads radially outwards and enters the $i$ th internal boundary-layer $(L)$ element (see Fig. 1).

Similarly, for the $i$ th internal boundary-layer $(L)$ element (see Fig. 1)

$$
\begin{aligned}
\dot{m}_{i, L}^{(\lambda)} & =J_{i-1, L}^{(\lambda)}-J_{i, L}^{(\lambda)}+J_{i, B L}^{(\lambda)} \\
\frac{d}{d t} \sum_{\lambda=v, g} m_{i, L}^{(\lambda)} u_{i, L}^{(\lambda)} & =\dot{Q}_{i, e}^{(v)}-\dot{W}_{i, L}^{(v)}+\sum_{\lambda=v, g} J_{i-1, L}^{(\lambda)} h_{i-1, L}^{(\lambda)}-J_{i, L}^{(\lambda)} h_{i, L}^{(\lambda)}+J_{i, B L}^{(\lambda)} h_{i, B}^{(\lambda)}
\end{aligned}
$$

Here, the specific enthalpies and energies of the elements are proportional to their absolute temperatures:

$$
\begin{aligned}
& h_{i, L(B)}^{v(g)}=c_{P, v(g)} T_{i, L(B)} \\
& u_{i, L(B)}^{v(g)}=c_{V, v(g)} T_{i, L(B)}
\end{aligned}
$$

For the $i$ th layer, the power associated with the change in its volume, $V_{i, B(L)}[1,15]$ :

$$
\dot{W}_{i, B(L)}^{(v)}=\left(p_{v}+p_{g}\right) \dot{V}_{i, B(L)}^{(v)}
$$

The rate of the heat transfer from the wall to the vapor boundary layer element [15-21]

$$
\dot{Q}_{i, e}^{(v)}=\alpha_{i} A_{i}\left(T_{w, v}-T_{i, B}^{(v)}\right)
$$

where $A_{i}=2 \pi R \Delta_{i}$ is the lateral surface of the boundary-layer element of the height $\Delta_{i}$. (In this paper the tank wall in contact with vapor is treated as a separate $\mathrm{CV}$ with temperature $T_{w v}$ compared with the tank part in contact with LH2 which has temperature $T_{w l}$.)

In the case of the isothermal vertical wall (Fig. 2), the heat transfer coefficient can be calculated as $[7,8$, $16-21]$

$$
\alpha_{i}=\frac{\kappa}{x_{i}} \begin{cases}0.68+0.503\left(R a_{i} \Psi\right)^{1 / 4}, & 10^{5}<R a<10^{9} \\ 0.15(R a \Psi)^{1 / 3}, & 10^{9} \leq R a<10^{11}\end{cases}
$$




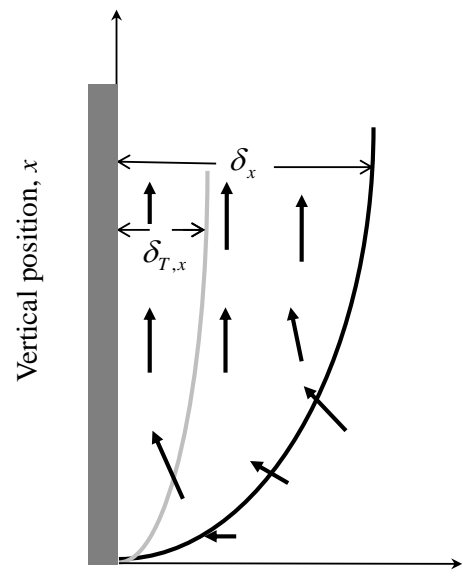

Distance to wall, $y$

(a)

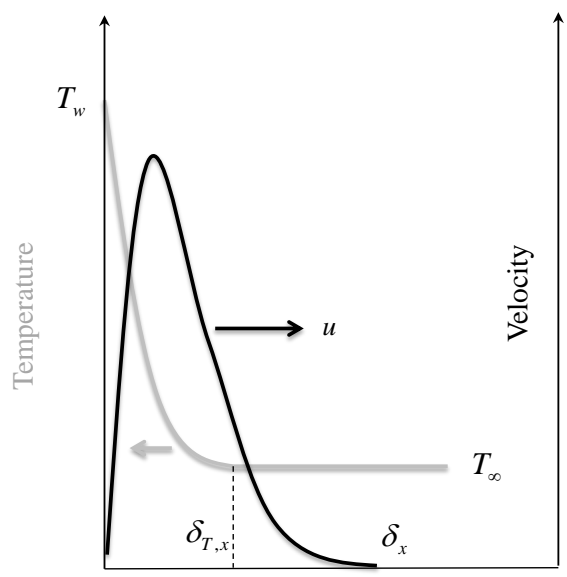

Distance to wall, $y$

(b)

Fig. 2 Natural convection from a vertical wall: (a) boundary layer; (b) temperature and velocity profiles.

where $x_{i}$ is the average elevation of the $i$ th layer counted from the interface (vapor film), $\kappa$ is the thermal conductivity of the GH2/GHe mixture, and

$$
\Psi=\left(1+\left(\frac{0.492}{\operatorname{Pr}}\right)^{9 / 16}\right)^{-16 / 9}
$$

Here the Raleigh number

$$
R a_{i}=G r_{i} \operatorname{Pr}
$$

and the Grashof number

$$
G r_{i}=\frac{g \beta\left(T_{w}-T_{i, B}\right) x_{i}^{3}}{\nu^{2}}
$$

The Prandtl number $\operatorname{Pr}=\mu c_{P} / \kappa$, where for an ideal gas the coefficient of the volumetric expansion $\beta=$ $1 / T_{i, L}$

The vertical mass flow rates within the $i$ th boundary layer $(i=1, \ldots, n-1)$ can be estimated as $[7,8]$

$$
J_{i, L}=2 \pi R \rho_{i} v_{i} \delta_{i} \begin{cases}0.0833, & \text { lam } \\ 0.1436, & \text { turb }\end{cases}
$$

where the average velocity of the vertical boundary-layer flow [16-21]

$$
v_{i}=1.185 \frac{\nu}{x_{i}}\left(\frac{G r_{i}}{1+0.494 \operatorname{Pr}^{2 / 3}}\right)^{1 / 2}
$$


and the thickness of the velocity boundary layer, within which the vertical component of the velocity is not very small (Fig. 2), can be calculated as [16-21]

$$
\delta_{i}=x_{i} \begin{cases}3.93\left(\frac{0.952+\operatorname{Pr}}{G r_{i} \operatorname{Pr}^{2}}\right)^{1 / 4}, & \text { lam } \\ 0.565\left(\frac{1+0.494 \operatorname{Pr}^{2 / 3}}{G r_{i}}\right)^{1 / 10} / \operatorname{Pr}^{8 / 15}, & \text { turb }\end{cases}
$$

It can be seen that the vertical mass flow rate is fully defined by the parameters of the corresponding boundary layer element $(i, L)$. In the case of natural convection, the mass flow is treated as laminar when the Raleigh number (11), which here plays a role of the Re number, is small [15], usually $R a<10^{9}$ [15-20]. The thickness of the thermal boundary layer, where the temperature changes from that of the wall $T_{w}$ to that of the bulk $T_{i, L}$

$$
\delta_{i, T}=\delta_{i} / \operatorname{Pr}^{1 / 2}
$$

is usually smaller than that of the velocity layer $(\operatorname{Pr}>1)$ [16-20] (see Fig. 2). In this paper the effects of film boiling are ignored [14]. A slight modification of the above formulas, describing the boundary layer effects, is required if the wall is not considered isothermal (see [15-21] and Appendix A).

For the lowest horizontal vapor layer $(i=1)$, which is in contact with the film CV,

$$
\begin{aligned}
\dot{m}_{1, B}^{(v)} & =J_{2, B}^{(v)}-J_{1, L}^{(v)}-J_{l v} \\
\dot{m}_{1, B}^{(g)} & =J_{2, B}^{(g)}-J_{1, L}^{(g)} \\
\frac{d}{d t} \sum_{\lambda=v, g} m_{1, B}^{(\lambda)} u_{1, B}^{(\lambda)} & =\dot{Q}_{v}-\dot{W}_{1, B}^{(v)}+\sum_{\lambda=v, g}\left(J_{2, B}^{(\lambda)} h_{2, B}^{(\lambda)}-J_{1, L}^{(\lambda)} h_{1, L}^{(\lambda)}\right)-J_{l v} h_{v s}
\end{aligned}
$$

Here, it is assumed that the lowest horizontal layer does not have the boundary element, so that the horizontal flow is equal to the upward vertical flow $(1, L)$ and is given by (13).

In accordance with [1] the rate of the heat exchange between the lowest horizontal gaseous layer and the vapor interface film $\mathrm{CV}$, which is driven either by convection or by conduction, can be calculated as

$$
\dot{Q}_{v}= \pm \max \left\{\dot{Q}_{v}^{\text {cond }}, \dot{Q}_{v}^{\text {conv }}\right\}
$$

where + is chosen when the temperature of the vapor-liquid interface, $T_{f}=T_{S}<T_{1, B}^{(v)}$, and - otherwise; the rate of the heat exchange due to convection

$$
\dot{Q}_{v}^{c o n v}=\pi R^{2} \alpha_{h o r}^{(v)}\left(T_{S}-T_{1, B}^{(v)}\right) H\left(T_{S}-T_{1, B}^{(v)}\right)
$$


where the Heaviside function $H$ takes care of the proper temperature condition required for convection; the heat transfer coefficient from a horizontal surface [16-20]

$$
\alpha_{h o r}=\frac{\kappa}{R} \begin{cases}0.54 R a_{R}^{1 / 4}, & 10^{4}<R a_{R}<10^{7} \\ 0.15 R a_{R}^{1 / 3}, & 10^{7} \leq R a_{R}<10^{11}\end{cases}
$$

Here the $R a_{R}$ number is given by (11) and (12) where $T_{w}-T_{i, B}$ should be substituted with $T_{s}-T_{1, B}$ and $x$ with $R$; and $\Psi$ is given by (10). In (20) the rate of the heat exchange due to conduction, $\dot{Q}_{v}^{c o n d}$, when it dominates heat flow through the interface, is defined by the time evolution of the temperature of the interface (film) $T_{S}(t)$. All the details of computation of $\dot{Q}_{v}^{c o n d}$ can be found in our previous paper [1].

For the upper horizontal layer, which is in contact with the top of the tank and presumably with the vent valve,

$$
\begin{aligned}
\dot{m}_{n, B}^{(\lambda)} & =J_{n-1, L}-J_{n, B}^{(\lambda)}+J_{\lambda, e} \\
\frac{d}{d t} \sum_{\lambda=v, g} m_{n, B}^{(\lambda)} u_{n, B}^{(\lambda)} & =\dot{Q}_{t o p}-\dot{W}_{n, B}^{(v)}+\sum_{\lambda=v, g}\left(J_{n-1, L}^{(\lambda)} h_{n-1, L}^{(\lambda)}-J_{n, B}^{(\lambda)} h_{n, B}^{(\lambda)}+J_{\lambda, e}\left(h_{n, B}^{(\lambda)}+v_{\lambda, e}^{2} / 2\right)\right)
\end{aligned}
$$

where the rate of the heat exchange with the top of the tank is given by

$$
\dot{Q}_{t o p}=\pi R^{2} \alpha_{h o r}^{(v)}\left(T_{w v}-T_{n, B}^{(v)}\right)
$$

with the heat transfer coefficient given by (22) where $T_{S}-T_{1, B}$ should be substituted with $T_{w v}-T_{n, B}^{(v)}$. Here, it is assumed that the upper horizontal layer does not have the boundary element (see Fig. 1).

\section{B. Liquid Control Volume}

Divide the liquid CV into $k$ horizontal layers, similar to the vapor CV. (The total number of the liquid $\mathrm{CVs}$ is equal to $2(k-1)$.) Then for the $j$ th bulk element $(j=2, \ldots, k-1)$; the levels are counted from the bottom of the tank up to the liquid/vapor interface)

$$
\begin{aligned}
\dot{m}_{j, B}^{(l)} & =J_{j+1, B}^{(l)}-J_{j, B}^{(l)}-J_{j, B L}^{(l)} \\
\frac{d}{d t} m_{j, B}^{(l)} u_{j, B}^{(l)} & =-\dot{W}_{j, B}^{(l)}+J_{j+1, B}^{(l)} h_{j+1, B}^{(l)}-\left(J_{j, B}^{(l)}+J_{j, B L}^{(l)}\right) h_{j, B}^{(l)}
\end{aligned}
$$


For the $j$ th internal boundary-layer $(L)$ liquid element, the mass and energy conservation, respectively, yield (see Fig. 1)

$$
\begin{aligned}
\dot{m}_{j, L}^{(l)} & =J_{j-1, L}^{(l)}-J_{j, L}^{(l)}+J_{j, B L}^{(l)} \\
\frac{d}{d t} m_{j, L}^{(l)} u_{j, L}^{(l)} & =\dot{Q}_{j, e}^{(l)}-\dot{W}_{j, L}^{(l)}+J_{j-1, L}^{(l)} h_{j-1, L}^{(l)}-J_{j, L}^{(l)} h_{j, L}^{(l)}+J_{j, B L}^{(l)} h_{j, B}^{(l)}
\end{aligned}
$$

Here the specific enthalpy and energy are close to each other and both approximately equal to [1]:

$$
h_{j, L(B)} \approx u_{j, L(B)} \approx c_{l} T_{j, L(B)}
$$

For the upper horizontal layer, which is in contact with the film CV (interface),

$$
\begin{aligned}
\dot{m}_{k, B}^{(l)} & =-J_{k, B}^{(l)}+J_{k-1, L}^{(l)}+J_{l v} \\
\frac{d}{d t} m_{k, B}^{(l)} u_{k, b}^{(l)} & =\dot{Q}_{l}-\dot{W}_{k, B}^{(l)}-J_{k, B}^{(l)} h_{k, b}^{(l)}+J_{k-1, L}^{(l)} h_{k-1, L}^{(l)}+J_{l v} h_{l s}
\end{aligned}
$$

Here it is assumed that the lowest horizontal layer does not have the boundary element, so that the upward vertical flow is equal to the horizontal flow and is given by (13).

Here, the rate of the heat exchange with the film CV

$$
\dot{Q}_{l}= \pm \max \left\{\dot{Q}_{l}^{\text {cond }}, \dot{Q}_{l}^{c o n v}\right\}
$$

where + is chosen when $T_{S}>T_{k, B}^{(l)}$, and - otherwise; the rate of the heat exchange due to convection

$$
\dot{Q}_{l}^{c o n v}=\pi R^{2} \alpha_{h o r}^{(l)}\left(T_{k, B}^{(l)}-T_{S}\right) H\left(T_{k, B}^{(l)}-T_{S}\right)
$$

with the heat transfer coefficient given by Eq. (22) with parameters corresponding to LH2 [15-21].

For the lowest horizontal layer, which is in contact with the bottom of the tank, and, assumingly with a transfer line valve

$$
\begin{aligned}
\dot{m}_{1, B}^{(l)} & =-J_{1, B}^{(l)}+J_{2, B}^{(l)}-J_{l, e} \\
\frac{d}{d t} m_{1, B}^{(l)} u_{1, B}^{(l)} & =\dot{Q}_{b o t t}-\dot{W}_{1, B}^{(l)}-J_{1, B}^{(l)} h_{1, B}^{(l)}+J_{2, L}^{(l)} h_{2, L}^{(l)}-J_{l, e}\left(h_{1, B}^{(l)}+v_{l, e}^{2} / 2\right)
\end{aligned}
$$

where the rate of the heat exchange with the bottom

$$
\dot{Q}_{b o t t}=\pi R^{2} \alpha_{h o r}^{(l)}\left(T_{w l}-T_{1, B}^{(l)}\right)
$$

with the heat transfer coefficient given by (22) where $T_{w v}-T_{1, B}^{(v)}$ should be substituted with $T_{w l}-T_{1, B}^{(l)}$. Here, it is assumed that the lowest liquid horizontal layer does not have the boundary element. (All the details of computation of the heat flow due to conduction, $\dot{Q}_{l}^{\text {cond }}$ can be found in our previous paper [1].) 


\section{Summary of the Model}

Thus, dividing the liquid $\mathrm{CV}$ into $k$ horizontal layers and the vapor $\mathrm{CV}$ into $n$ layers, one deals with the following $2(5 n+3 k)-13$ state variables: $4(n-1)+2(k-1)=2(2 n+$ $k-3)$ masses $m_{1, B}^{(\lambda)}, m_{n, B}^{(\lambda)}, m_{i, B(L)}^{(\lambda)}, m_{1, B}^{(l)}, m_{k, B}^{(l)}, m_{j, B(L)}^{(l)}(i=2, \ldots, n-1 ; j=2, \ldots, k-$ $1 ; \lambda=v, g)$; vapor $\mathrm{CV}$ partial pressures and volume $p_{v}, p_{g}$ and $V_{v} ; 2(n+k)-1$ temperatures $T_{f}, T_{w l}, T_{w v}, T_{1, B}^{(v)}, T_{n, B}^{(v)}, T_{i, B(L)}^{(v)}, T_{1, B}^{(l)}, T_{k, B}^{(l)}, T_{j, B(L)}^{(l)}(i=2, \ldots, n-1 ; j=2, \ldots, k-1) ;$ and $4(n-2)+$ $2(k-2)+3=2(2 n+k)-9$ mass flow rates $J_{i, B}^{(\lambda)}, J_{i, B L}^{(\lambda)}, J_{n, B}^{(\lambda)}, J_{j, B}^{(l)}, J_{j, B L}^{(l)}, J_{1, B}^{(l)}(i=2, \ldots, n-1 ; j=$ $2, \ldots, k-1 ; \lambda=v, g)$

There are $4 n+2 k-5$ constraints: $4(n-1) \mathrm{GH} 2$ and GHE equations of state;

$$
p_{\lambda} V_{i, B(L)}^{(v)}=m_{i, B(L)}^{(\lambda)} R_{\lambda} T_{i, B(L)}^{(v)}
$$

where $\lambda=v, g$; the film equation of state

$$
p_{v}=p_{f}\left(T_{f}\right)=p_{C}\left(T_{f} / T_{C}\right)^{n}
$$

with $p_{C}=1.315 \mathrm{MPa}$ and $T_{C}=33.2 \mathrm{~K}$ and $n=5$ being, respectively, critical pressure and temperature for hydrogen [1] (in (38), the partial pressures are considered uniform across the vapor CV, with the LH2 partial pressure equal to that of the saturated vapor film (39); $2(k-1)-1$ equations relating the liquid masses and volumes (because one liquid mass is fully defined by the total liquid mass, which, in turn is defined by (41))

$$
m_{j, L(B)}^{(l)}=\rho_{l} V_{j, L(B)}^{(l)}
$$

and an equation that relates the total tank volume to the $\mathrm{GH} 2$ volume and $\mathrm{LH} 2$ volume

$$
V=V_{v}+V_{l}=\left(V_{1, B}^{(v)}+V_{n, B}^{(v)}+\sum_{i=2}^{n-1}\left(V_{i, B}^{(v)}+V_{i, L}^{(v)}\right)\right)+\left(V_{1, B}^{(l)}+V_{k, B}^{(l)}+\sum_{j=2}^{k-1}\left(V_{j, B}^{(l)}+V_{j, L}^{(l)}\right)\right)
$$

There are $6 n+4 k-4$ ordinary differential rate equations for $2(5 n+3 k)-13-(4 n+2 k-9)=6 n+4 k-4$ independent variables: $4(n-1) \mathrm{GH} 2$ and GHe mass conservation equations (1), (3), (17), (18), and (23); $4(n-1) \mathrm{GH} 2$ and GHe energy conservation equations (2), (4), (19), and (24); $2(k-1)$ LH2 mass conservation equations (26), (28), (31), and (35); 2(k-1) LH2 energy conservation equations (27), (29), (32), and (36); and two energy conservation equations for the two control volumes for the tank wall in contact with LH2 and 


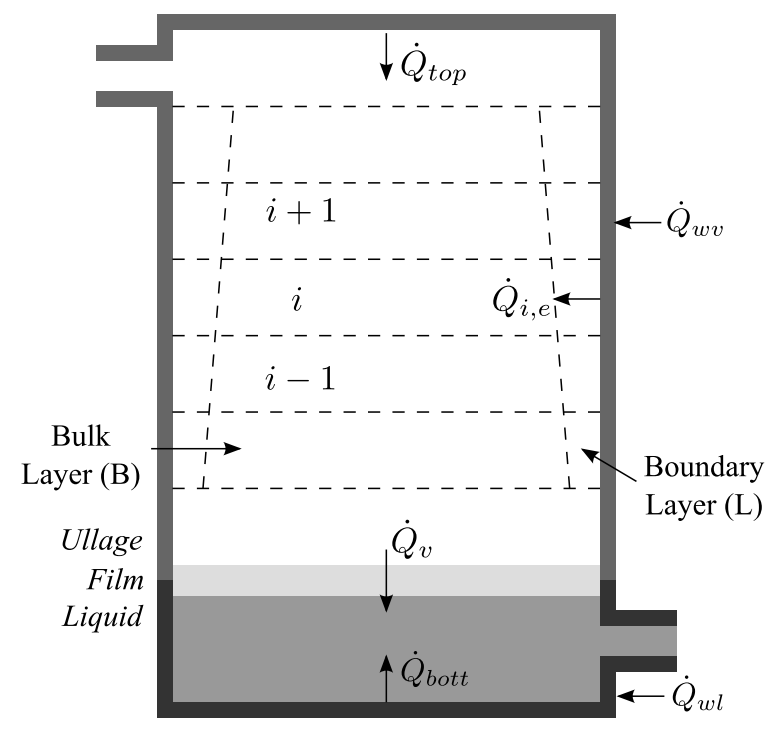

(a) Side view.

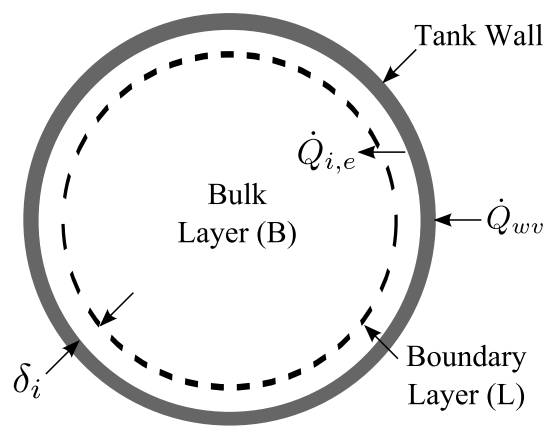

(b) Top view.

Fig. 3 Heat flow in a propellant tank with three control volumes (vapor, film, and liquid).

the ullage, respectively (Fig. 3)

$$
\begin{gathered}
c_{w} d\left(m_{w l} T_{w l}\right) / d t= \begin{cases}\dot{Q}_{w l}-\dot{Q}_{e}^{(l)}+c_{w} \dot{m}_{w l} T_{w l}, & \dot{m}_{w l}<0 \\
\dot{Q}_{w l}-\dot{Q}_{e}^{(l)}+c_{w} \dot{m}_{w l} T_{w v}, & \dot{m}_{w l} \geq 0\end{cases} \\
c_{w} d\left(m_{w v} T_{w v}\right) / d t= \begin{cases}\dot{Q}_{w v}-\dot{Q}_{e}^{(v)}+c_{w} \dot{m}_{w v} T_{w l}, & \dot{m}_{w l}<0 \\
\dot{Q}_{w v}-\dot{Q}_{e}^{(v)}+c_{w} \dot{m}_{w v} T_{w v}, & \dot{m}_{w l} \geq 0\end{cases}
\end{gathered}
$$

Here the last terms in the right sides of Eqs. 42 and 43 describe the flow of internal energy along the tank walls due to motion of the liquid level and of the subsequent plane separating the two tank CVs (note that $\dot{m}_{w l}=-\dot{m}_{w v}$ ). Also, in the above equations we ignore the direct heat exchange (due to conduction) between the two parts of the tank because the corresponding thermal length even for $t=10^{3} \mathrm{~s}$ is shown to be much smaller than the size of those parts. The total heat transfer from the tank wall to the vapor CV (see (8) and $(25))$

$$
\dot{Q}_{e}^{(v)}=\dot{Q}_{t o p}+\sum_{i=2}^{n-2} \dot{Q}_{i, e}^{(v)}
$$

and the total heat transfer from the tank wall to the liquid CV

$$
\dot{Q}_{e}^{(l)}=\dot{Q}_{b o t t}+\sum_{j=2}^{k-2} \dot{Q}_{j, e}^{(l)}
$$


where

$$
\dot{Q}_{j, e}^{(l)}=\alpha_{j} A_{j}\left(T_{w l}-T_{j, B}^{(l)}\right)
$$

and $\dot{Q}_{b o t t}$ is given by (37). Here the heat transfer coefficients $\alpha_{j}$ are given by (8) with the parameters of liquid and $A_{j}=2 \pi R \Delta_{j}$ is the lateral surface of the boundary-layer element of the height $\Delta_{j}$.

And, finally, the condensation-evaporation mass flow rate through the LH2/GH2 interface, $J_{l v}$, is related to the heat flows through the same interface, $\dot{Q}_{v}(20)$ and $\dot{Q}_{l}(33)$ by means of the energy conservation equation for the film CV. Ignoring the film mass, it can be written as

$$
\dot{Q}_{v}-\dot{Q}_{l}+J_{l v}\left(h_{v s}-h_{l s}\right)=\frac{d}{d t} m_{f} u_{f}=0
$$

where the specific enthalpies are referred to those of the saturated vapor and liquid. The temperatures of the vapor liquid interface, $T_{S}$, is considered to be equal of that of the film $T_{f}$ [1].

\section{Results}

The above described model of temperature stratification has been explored in an LH2 tank. The parameter values of the cylindrical tank were chosen similar to that of the Space Shuttle ET [1, 2], provided in Appendix C. In Eqs. 42 and 43, the material parameters of the tank are chosen to that typical for aluminum (see Table 1 in Appendix C). The tank is considered to be initially (at $t=0$ ) half-filled with LH2: $V_{l}(0)=V / 2$ with GH2 and GHe partial pressures $p_{v}(0)=p_{g}(0)=1$ atm and initial temperatures of GH2/GHe mixture, $\mathrm{LH} 2$, and the tank, respectively equal to $T_{v}(0)=T_{l}(0)=20 \mathrm{~K}$ and $T_{w}(0)=21 \mathrm{~K}$. In addition, the tank absorbs heat from the environment (see Eqs. 42 and 43) at a rate of $\dot{Q}_{w}=20 \mathrm{~kW}$ in the blowdown regime. Throughout modeling, the number of horizontal layers in the liquid CV was set equal to that in the vapor $\mathrm{CV}$, where $n=k=10$, which yields 146 state variables and 95 ordinary differential equations. It is found that starting from $n=k=10$ and on, such "integral" characteristics as the total ullage pressure $p_{v}+p_{g}$ and the tank wall temperatures $T_{w l}$ and $T_{w v}$ converge and change no more than, respectively, $0.1 \%$ and $0.3 \%$ with the increase of the number of the horizontal layers. Those numbers can be taken as a measure of the uncertainty of the predicted results. 


\section{A. Blowdown Regime}

In the blowdown regime it is assumed that the ullage vent valve is closed $\left(J_{v, e}=J_{g, e}=0\right)$, and LH2 is being removed from the tank at a constant rate of $J_{l, e}=10 \mathrm{~kg} / \mathrm{s}$. The case of normal gravity is considered first. Fig. 4 shows the masses and temperatures. (For this and all the subsequent figures higher layers are shown with lighter-colored lines.) LH2 mass (Fig. 4a) drops, due to the positive external mass flow, but also due to evaporation, while the GH2 mass (Fig. 4b) increases due to evaporation. The very same evaporation is responsible for cooling off the $\mathrm{LH} 2 / \mathrm{GH} 2$ interface, and the liquid-vapor interface temperature $T_{S}=T_{f}$ drops due to the evaporation ((Figs. 4c and 4e). The lumped-parameter ullage temperature is also shown in Fig. 4e as $\bar{T}_{v}$ to demonstrate that the stratified temperatures behavior is consistent with the lumped-parameter formulation (see Appendix B). The expansion of the ullage volume leads to its initial cooling, which gives way to the subsequent ullage heating due to effective convection-assisted heat transfer from the tank wall (Fig. 4e). The temperatures of the liquid layers also increase due to heat transfer from the tank wall, as shown in Fig. 4c. Fig. 4d shows only the temperatures of the liquid bulk temperatures, since the stratification is not easily observed in Fig. 4c. Fig. 5 uses a heat map to show a visualization of temperature stratification in this case at different time instants.

Fig. 6 demonstrates temperature evolution of different layers of the tank as it would be measured by sensors at fixed heights in the tank. Here, $n=100$ is used to minimize an artificial "ripple" effect caused by transition from one layer to another that happens at a fixed sensor height. It can be seen that as the liquid level crosses the sensor at $12 \mathrm{~m}$, the temperature experiences a sudden drop from the LH2 temperature to that of the GH2/GHe mixture. A small peak seen at the ullage temperature curves can be attributed to transient effects that develop at the early stages of the system evolution.

Fig. 7 shows the masses and temperatures for the blowdown regime in elevated gravity $(7 g)$. Here, the evaporation rate is somewhat larger than the case of normal gravity (compare Figs. 4b and 7b), and stratification seems to be reduced slightly due to the enhanced convection-driven heat exchange between the tank body and its contents (see Figs. 7c, 7d, and 7e). Fig. 8 shows the visualization of temperature stratification in this case.

Fig. 9 shows behavior of the masses and temperatures for the tank at the conditions of microgravity $\left(10^{-6} g\right)$. Here, the heat exchange between the tank wall and its contents is suppressed, thus leading to 


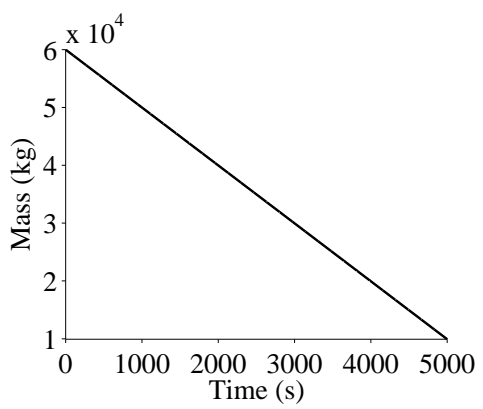

(a) LH2 Mass

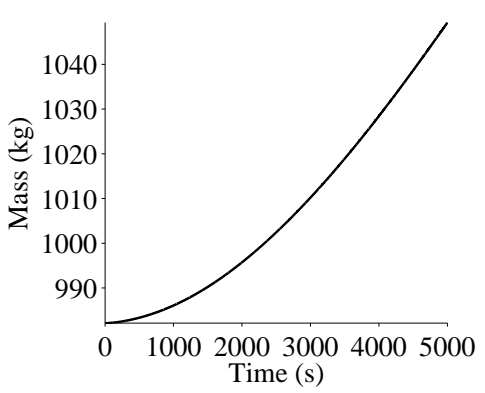

(b) GH2 Mass

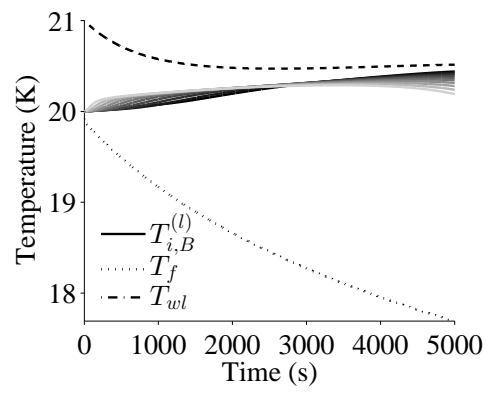

(c) LH2 Temperatures

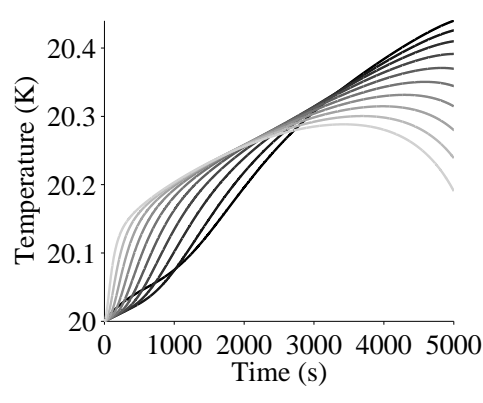

(d) LH2 Bulk Temperatures

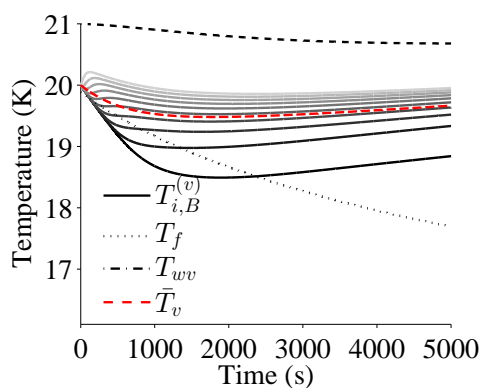

(e) GH2/GHe Temperatures

Fig. 4 Tank in a blowdown regime at normal gravity.

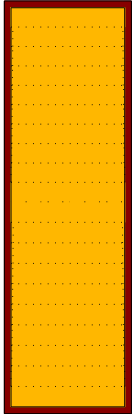

(a) $t=0 \mathrm{~s}$

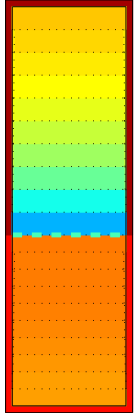

(b) $t=1000 \mathrm{~s}$

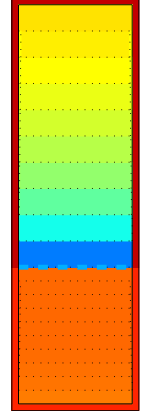

(c) $t=2000 \mathrm{~s}$

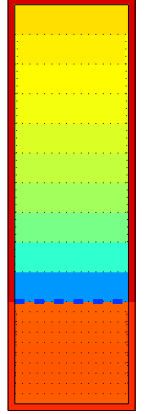

(d) $t=3000 \mathrm{~s}$

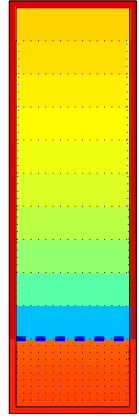

(e) $t=4000 \mathrm{~s}$

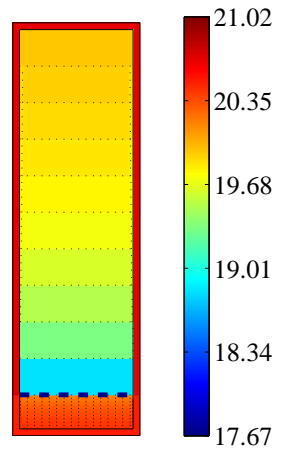

(f) $t=5000 \mathrm{~s}$

Fig. 5 Tank in a blowdown regime at normal gravity: temperature stratification.

continuous cooling of the ullage due to its expansion. In this case, the suppression of the heat exchange, in contrast to the initial drop in the tank's temperature $T_{w l}$ and $T_{w v}$ at normal (Fig. 4) and elevated gravity (Fig. 7), causes continuous heating of the tank wall and diminishes temperature stratification effects in both the liquid and vapor CVs. This is clearly shown visualization of the temperature stratification in Fig. 10.

It should be mentioned here that in all cases, as expected, the temperature stratification is much more pronounced in the ullage $\mathrm{CV}$ compared to the liquid $\mathrm{CV}$ due to much more effective convection flow and 


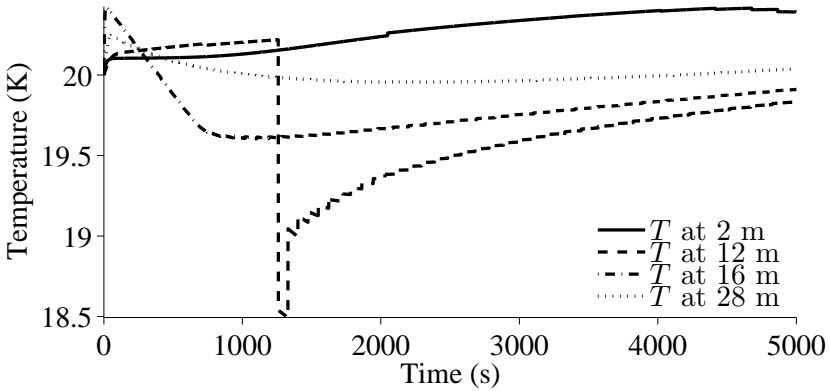

Fig. 6 Temperatures at fixed heights in a tank in a blowdown regime at normal gravity.

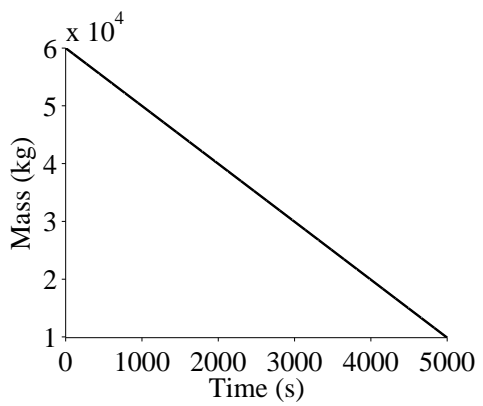

(a) LH2 Mass

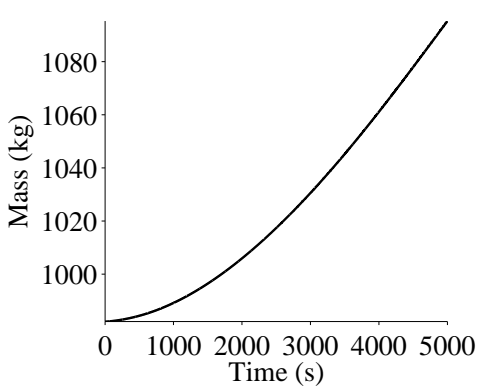

(b) GH2 Mass

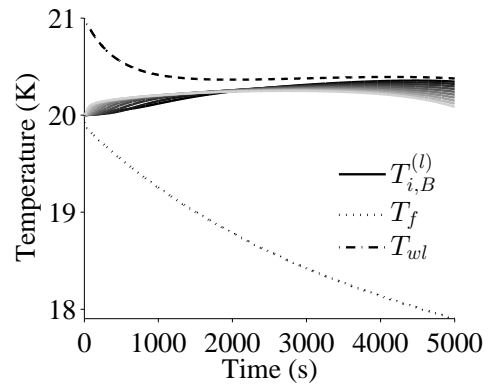

(c) LH2 Temperatures

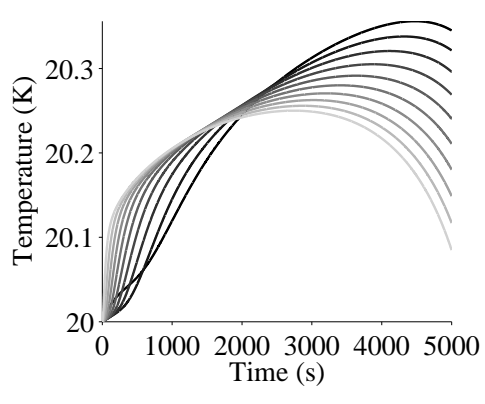

(d) LH2 Bulk Temperatures

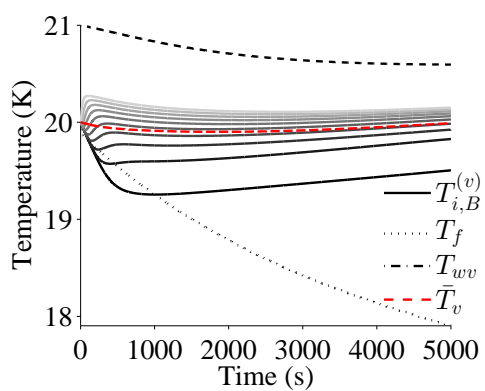

(e) GH2/GHe Temperatures

Fig. 7 Tank in a blowdown regime at elevated gravity $7 g$.

thermal conductivity in the LH2 $\left(J_{L}=5 \mathrm{~kg} / \mathrm{s}\right.$ in comparison with GH2/GHe where $\left.J_{L}=0.9 \mathrm{~kg} / \mathrm{s}\right)$. Figs. $4 \mathrm{e}$, $7 \mathrm{e}$, and $9 \mathrm{e}$ also demonstrate that the results of the simulations, which take into consideration the effects of temperature stratification, are fully consistent with previous ones (seen on $T_{v}$ curves) that are obtained in the framework of the lumped parameter method (see $[1,2]$ and Appendix B).

\section{B. Storage Regime}

The storage regime is now considered, where liquid is not added or removed from the tank $\left(J_{l, e}=0\right)$. The tank, with initial temperature set at $T_{w}(0)=20.3 \mathrm{~K}$ is absorbing heat at a rate of $\dot{Q}_{w}=10 \mathrm{~kW}$ that, for 


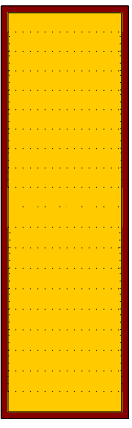

(a) $t=0 \mathrm{~s}$

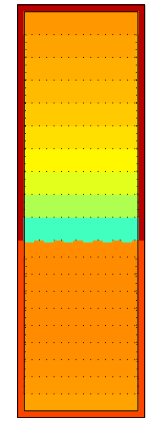

(b) $t=1000 \mathrm{~s}$

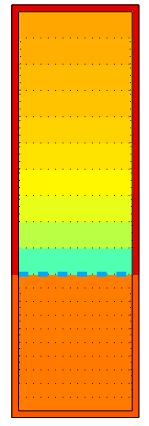

(c) $t=2000 \mathrm{~s}$

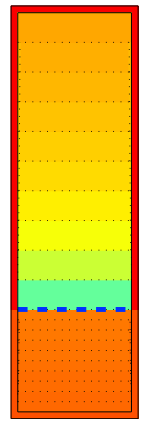

(d) $t=3000 \mathrm{~s}$

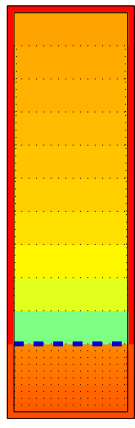

(e) $t=4000 \mathrm{~s}$

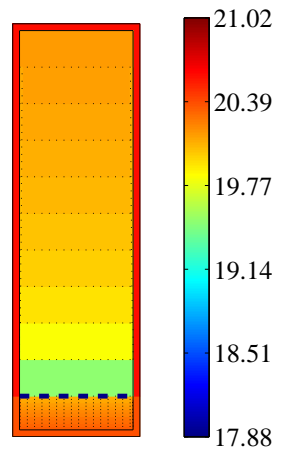

(f) $t=5000 \mathrm{~s}$

Fig. 8 Tank in a blowdown regime at elevated gravity: temperature stratification.

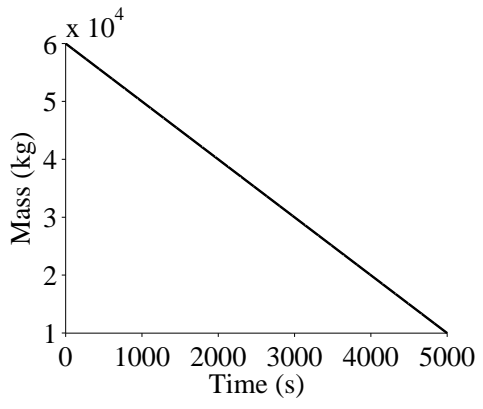

(a) LH2 Mass

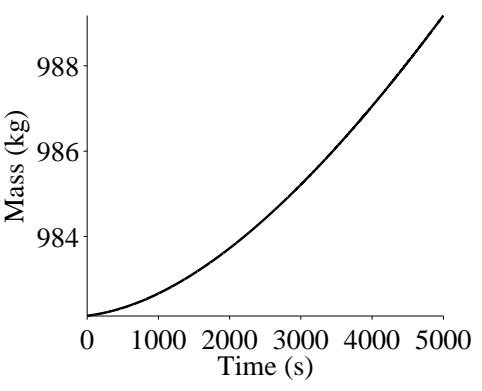

(b) GH2 Mass

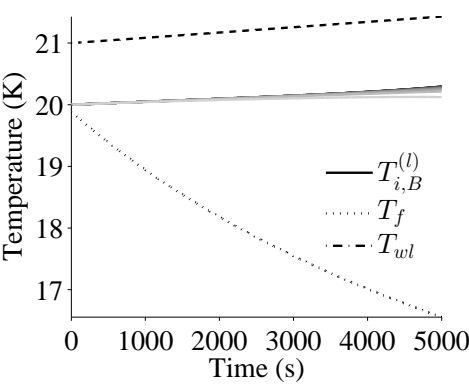

(c) LH2 Temperatures

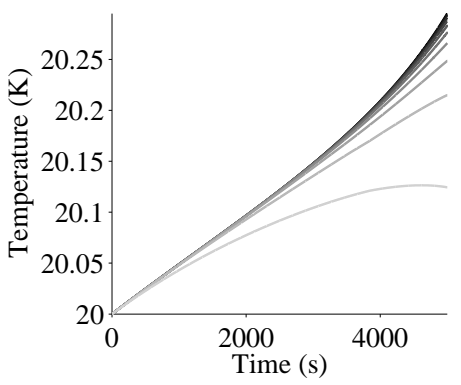

(d) LH2 Bulk Temperatures

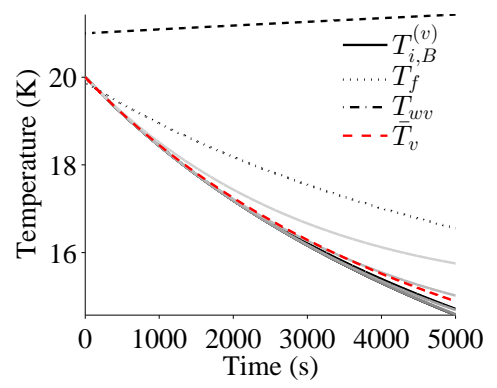

(e) GH2/GHe Temperatures

Fig. 9 Tank in a blowdown regime at microgravity.

the tank of a chosen size, roughly corresponds to the radiation heat flux from the Sun. Fig. 11 demonstrates temperature evolutions in the unvented tank $\left(J_{v, e}=J_{g, e}=0\right)$ at the conditions of normal gravity. Here, evaporation is greatly reduced (Fig. 11a). Substantial temperature stratification in the ullage volume can be observed. Also, it can be seen that in contrast to the blowdown regime (Fig. 4) all temperatures including that of the LH2/LG2 interface, $T_{f}$, rise because of continuing heat absorption. Here again,the interface temperature is less than that of $\mathrm{LH} 2$ due to evaporation. The temperature stratification visualization is shown 


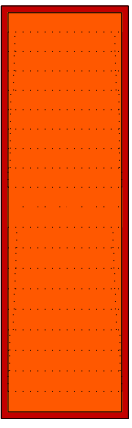

(a) $t=0 \mathrm{~s}$

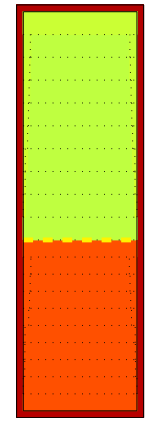

(b) $t=1000 \mathrm{~s}$

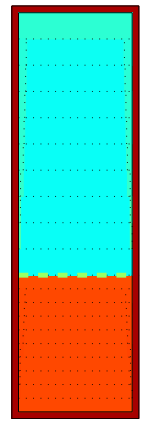

(c) $t=2000 \mathrm{~s}$

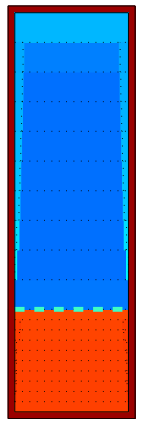

(d) $t=3000 \mathrm{~s}$

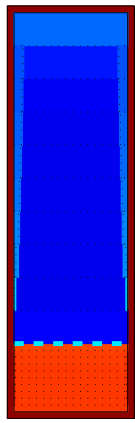

(e) $t=4000 \mathrm{~s}$

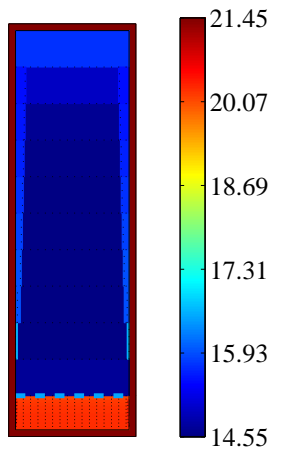

(f) $t=5000 \mathrm{~s}$

Fig. 10 Tank in a blowdown regime at microgravity: temperature stratification. (A slow development of the boundary layer can be seen at $t>4000$ s.)

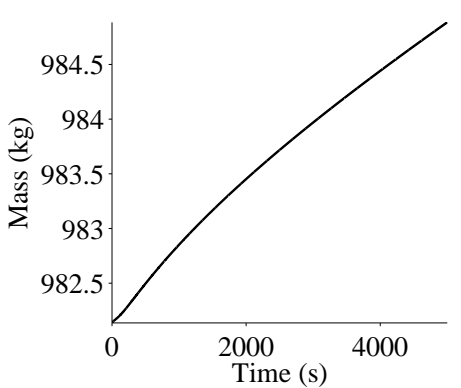

(a) GH2 Mass

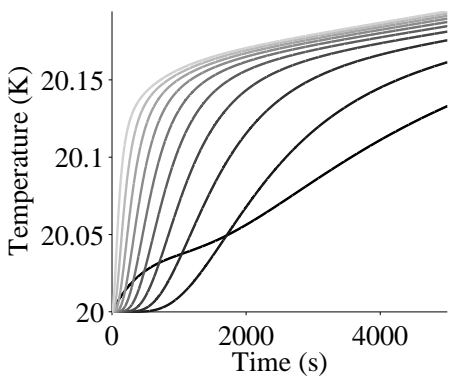

(c) LH2 Bulk Temperatures

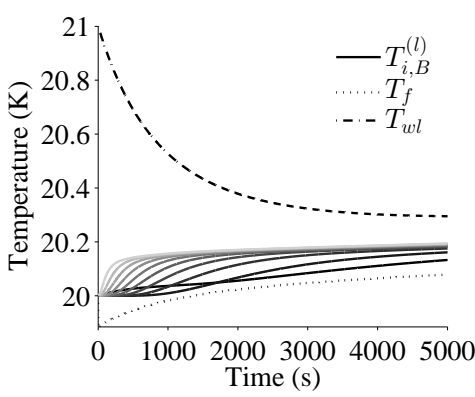

(b) LH2 Temperatures

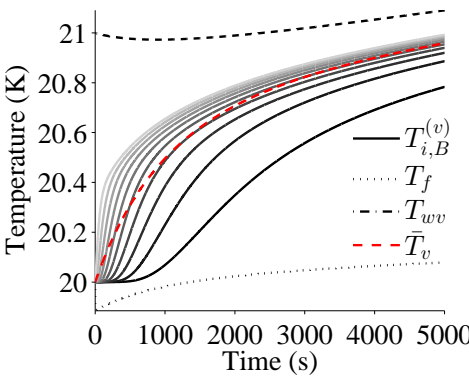

(d) GH2/GHe Temperatures

Fig. 11 Tank in a storage regime at normal gravity without venting.

in Fig. 12. Everything starts out very cold, and temperature stratification occurs naturally as heat is added to the tank wall. The ullage heats up while the liquid, due to its greater heat capacitance, stays relatively cool.

Introduction of a vent valve in the upper part of the ullage volume dramatically changes the picture, as shown in Fig. 13. The valve is set to operate between lower and upper thresholds at 2.00 and 2.02 atm, correspondingly. When the upper threshold is reached, the valve opens; it closes as soon as the lower limit is 


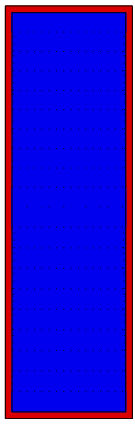

(a) $t=0 \mathrm{~s}$

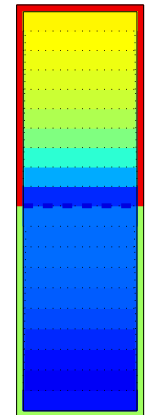

(b) $t=1000 \mathrm{~s}$

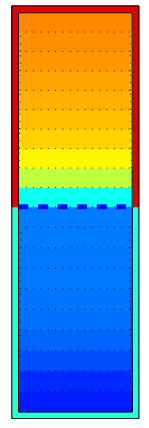

(c) $t=2000 \mathrm{~s}$

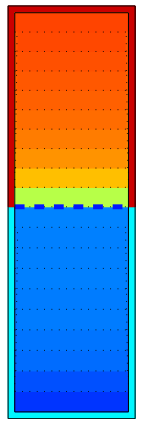

(d) $t=3000 \mathrm{~s}$

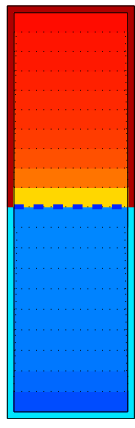

(e) $t=4000 \mathrm{~s}$

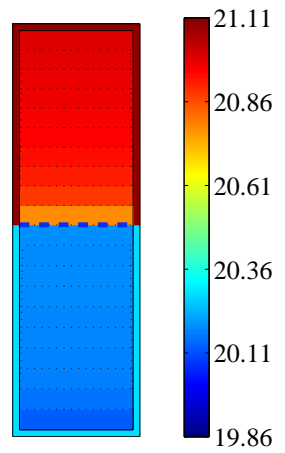

(f) $t=5000 \mathrm{~s}$

Fig. 12 Tank in a storage regime without venting at normal gravity: temperature stratification.

achieved. The gas flow rate through the vent valve $J_{v(g), e}=-J_{v a l v e}^{v(g)}$ where for the choked flow regime [22]

$$
J_{\text {valve }}^{v(g)}=\lambda \rho_{v(g)} S_{v, \text { valve }} \sqrt{\frac{\gamma\left(p_{v}+p_{g}\right)}{\rho_{v}+\rho_{g}}\left(\frac{2}{\gamma+1}\right)^{\frac{\gamma+1}{\gamma-1}}}
$$

Here $\lambda$ is a dimensionless relative valve position which assumes values between 0 and 1 . For a given composition of the GH2/GHe mixture, parameter $\gamma=1.51$, and the conditions for the choked flow regime [22] are satisfied. Also, in the present modeling, the valve's cross-section area is set as $S_{\text {valve }}=5 \mathrm{~cm}^{2}$. Each time the valve opens, a certain amount of the gas/vapor mixture from the upper layer is removed (Figs. 13b and 13c). Since that amount is small compared to the total ullage mass its removal does not substantially change the ullage pressure (Fig. 13a), which is uniform across the ullage volume and propagates very quickly (with the speed of sound). Yet the removed mass is comparable with that of the upper layer, where mass reduction, in accordance with the equation of state (38), leads to a temperature spike (Fig. 13f). A subsequent temperature wave can be observed propagating in the downward direction due to downward convection circulation in the bulk layers (visualized in Fig. 14), which diminishes in intensity as it travels downwards. As a result, on average the temperatures rise at a smaller rate compared to the non-vent case (Fig. 12), and the interface temperature is being stabilized (Fig. 13f). Note, that due to fast propagation of the pressure, the temperature variations in the lower layers follow that of pressure and are opposite in phase to those of the upper layers.

\section{Conclusions}

In the present paper, the dynamical model of a cryogenic fuel tank has been presented that incorporates effects of (i) temperature stratification driven by the natural convection in both the liquid and ullage control 


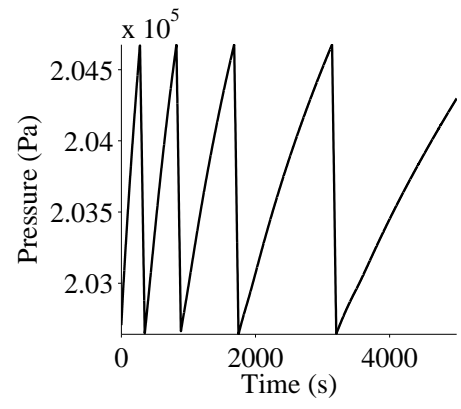

(a) Ullage Pressure

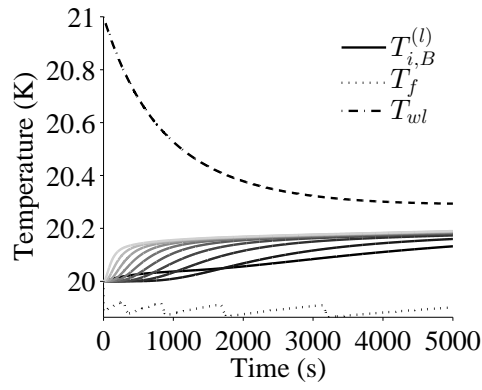

(d) LH2 Temperatures

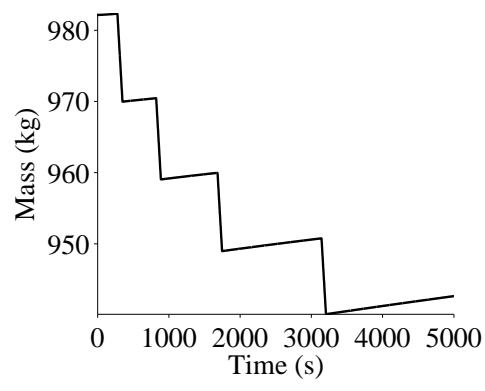

(b) GH2 Mass

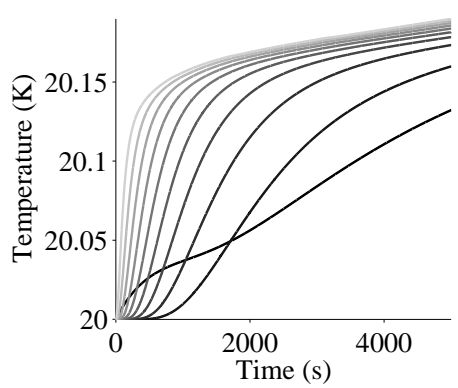

(e) LH2 Bulk Temperatures

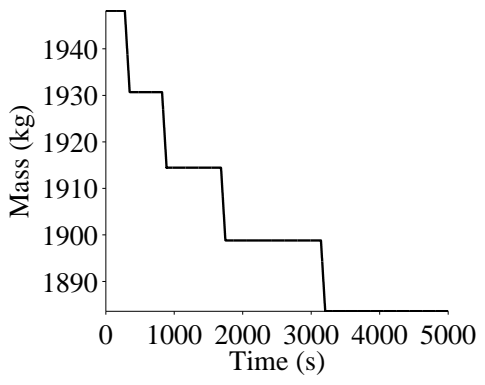

(c) GHe Mass

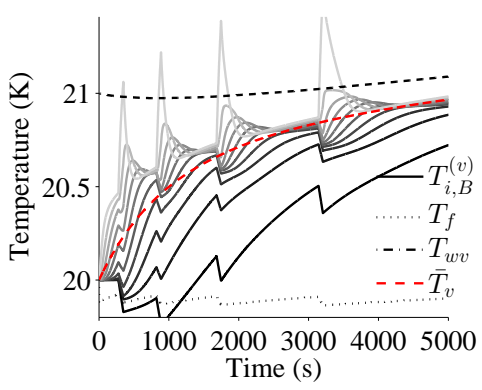

(f) $\mathrm{GH} 2 / \mathrm{GHe}$ Temperatures

Fig. 13 Tank in a storage regime at normal gravity with venting.

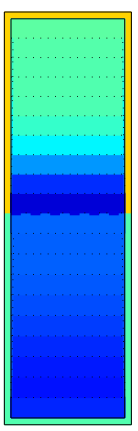

(a) $t=800 \mathrm{~s}$

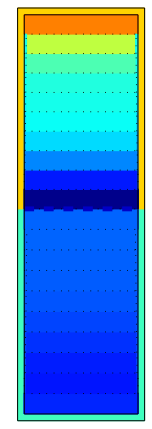

(b) $t=900 \mathrm{~s}$

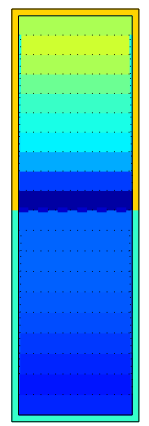

(c) $t=1000 \mathrm{~s}$

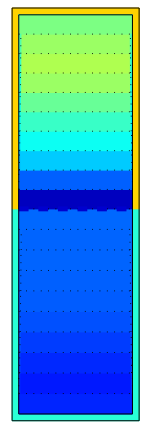

(d) $t=1100 \mathrm{~s}$

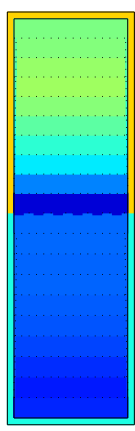

(e) $t=1200 \mathrm{~s}$

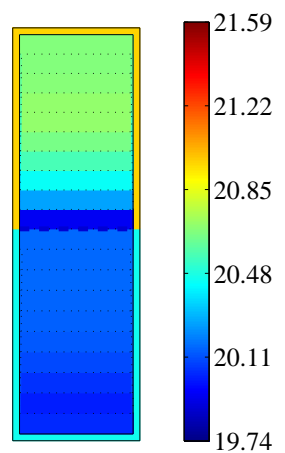

(f) $t=1300 \mathrm{~s}$

Fig. 14 Tank in a storage regime with venting at normal gravity: temperature stratification.

volumes and (ii) highly nonequilibrium condensation-evaporation phenomena at the interface between those volumes. Unlike standard full-scale computational fluid dynamics approach dealing with numerical solution of equations in partial derivatives, the proposed low-dimensional scheme reduces modeling to solution of a set of ordinary differential equations that account for basic conservation laws applied to the properly chosen control volumes. Those equations are demonstrated to successfully describe complex temperature stratification effects for different modes of the liquid hydrogen fuel tank operation. 
Firstly, it has been shown that the above effects are consistent with the description of the system in the framework of the lumped-parameter method. Secondly, time evolution of the temperature data collected by the fixed-elevation sensors can be described and analyzed in the framework of the developed approach. Thirdly, complex effects such as temperature waves caused by a vent valve operating between given pressure thresholds can be found and understood by means of the simplified computational approach. Fourthly, practically important and theoretically complex effects of normal, elevated and micro-gravity can be taken into consideration including their role in the heat exchange between the tank walls and the cryogenic fuel content. In particular, it has been demonstrated that the increased (decreased) gravity will substantially promote (suppress) such an exchange.

\section{Appendix A: Uniform Heat Flux}

In the above consideration, it is assumed that the tank is described by the uniform temperature of its body, $T_{w}$. In the case of the uniform heat flux ( $\dot{q}_{w}=c o n s t$ ), the local (average) Raleigh numbers $R a_{x}\left(R a_{H}\right)$ that define the heat transfer coefficients (9), (22) as well as the velocity (14) in, and the thickness (15) of, the boundary layers (via the Grashof numbers (12)), must be substituted by a modified Raleigh number [15]. It can be found by expressing Ra in terms of $\dot{q}_{w}$ by eliminating $T_{w}-T_{v(l)}$ :

$$
R a_{x}^{*}=R a_{x} N u_{x}=\frac{g \rho^{2} c_{P} \beta \dot{q}_{w} x^{4}}{\mu \kappa^{2}}
$$

so that the heat transfer coefficients in (9) are to be substituted with, correspondingly, the modified heat transfer coefficients [15]:

$$
\alpha_{i}=\frac{\kappa}{x_{i}} N u_{i}=\frac{\kappa}{x_{i}} \begin{cases}0.631\left(R a_{x}^{*} \Psi\right)^{1 / 5}, & 10^{5}<R a_{x}^{*}<10^{9} \\ 0.241\left(R a_{x}^{*} \Psi\right)^{1 / 4}, & 10^{9} \leq R a_{x}^{*}<10^{11}\end{cases}
$$

and

$$
\bar{\alpha}_{L}=\frac{\kappa}{H_{l}} N u_{H}=\frac{\kappa}{H_{l}} \begin{cases}0.726\left(R a_{H}^{*} \Psi\right)^{1 / 5}, & 10^{5}<R a_{H}^{*}<10^{9} \\ 0.241\left(R a_{H}^{*} \Psi\right)^{1 / 4}, & 10^{9} \leq R a_{H}^{*}<10^{11}\end{cases}
$$

where $\Psi$ is given by (10). Given the uniform heat flux $\dot{q}_{w}$ the local temperature at the wall surface can be found from

$$
T_{w}=T_{v(l)}+\frac{\dot{q}_{w}}{\alpha}=T_{v(l)}+\frac{\dot{q}_{w} x}{N u_{x} \kappa}
$$


where the $N u_{x}$ number can be found from (50).

\section{Appendix B: Lumped-parameter Approximation}

Here is described the lumped-parameter approximation for each of the three CVs: liquid, vapor and film, similar to that presented in [1] by ascribing a common temperature to each of those CVs. In that case the mass conservation equation for, e.g., the liquid CV can be obtained by summing up (26), (28), (31), and (35) over all liquid elements thus yielding

$$
\dot{m}_{l}=J_{l v}-J_{l, e}
$$

Here the interface flow rate $J_{l v}$ can be found by solving (7.1) together with the total-mass conservation for the vapor $\mathrm{CV}$

$$
\begin{aligned}
& \dot{m}_{v}=-J_{l v}+J_{v, e} \\
& \dot{m}_{g}=J_{g, e}
\end{aligned}
$$

and the energy conservation for the liquid, vapor and film CVs (see also (47)), all of which can be obtained in a similar way,:

$$
\begin{aligned}
\dot{Q}_{l, e}+\dot{Q}_{l}+\dot{W}+J_{l v} h_{l s}+J_{l, e}\left(h_{l}+v_{l, e}^{2} / 2\right) & =\frac{d}{d t} m_{l} u_{l} \\
\dot{Q}_{v, e}-\dot{Q}_{v}-\dot{W}-J_{l v} h_{v s}+J_{v, e}\left(h_{v} v_{v, e}^{2} / 2\right)+J_{g, e}\left(h_{g}+v_{g, e}^{2} / 2\right) & =\frac{d}{d t}\left(m_{v} u_{v}+m_{g} u_{g}\right)
\end{aligned}
$$

complemented with the expression relating the liquid and vapor volumes

$$
V=V_{v}+V_{l}=V_{v}+m_{l} / \rho_{l}
$$

and the film (39) and vapor(gas) equations of state

$$
p_{v(g)}=\rho_{v(g)} R_{v(g)} T_{v}
$$

Given the external mass flow rates, $J_{v, e}, J_{l, e}$, Eqs (53) - (59), (39), and (47) allow one to find the rate of the total mass change $\dot{m}_{l}$ (or $\dot{V}_{l}$ ) for the liquid $\mathrm{CV}$, the rate of the volume change $\dot{V}_{v}$ of the vapor $\mathrm{CV}$, as well as the "average" temperatures $T_{l}, T_{v}$ as functions of time. In this step, the local convection heat transfer 
coefficients for the vertical surfaces should be substituted by their average values [15]

$$
\bar{\alpha}_{H}=\frac{\kappa}{H_{l(v)}} \begin{cases}0.68+0.67\left(R a_{H} \Psi\right)^{1 / 4}, & 10^{5}<R a_{H}<10^{9} \\ 0.15\left(R a_{H} \Psi\right)^{1 / 3}, & 10^{9} \leq R a_{H}<10^{11}\end{cases}
$$

where $H_{l(v)}$ is the height of the liquid/vapor in contact with a vertical wall.

\section{Appendix C: Model Parameters}

Model parameter values are given in Table 1 . Here, $d_{w}$ refers to the tank wall thickness.

Table 1 Parameter Values

\begin{tabular}{ll}
\hline Component & Parameter Values \\
\hline Hydrogen and Helium & $T_{c}=33.2 \mathrm{~K}, p_{c}=1.315 \times 10^{6} \mathrm{~Pa}, \lambda=5, \rho_{L}=71.1 \mathrm{~kg} / \mathrm{m}^{3}, c_{L}=9450 \mathrm{~J} / \mathrm{kg} / \mathrm{K}$, \\
& $\kappa_{L}=0.0984 \mathrm{~W} / \mathrm{m} / \mathrm{K}, \mu=3.4 \times 10^{-6} \mathrm{~Pa} \mathrm{~s}, R_{v}=4124 \mathrm{~J} / \mathrm{kg} / \mathrm{K}$, \\
& $c_{V, v}=6490 \mathrm{~J} / \mathrm{kg} / \mathrm{K}, \gamma=5 / 3, \kappa_{v}=0.0166 \mathrm{~W} / \mathrm{m} / \mathrm{K}, R_{g}=2077 \mathrm{~J} / \mathrm{kg} / \mathrm{K}$, \\
& $\kappa_{g}=0.0262 \mathrm{~W} / \mathrm{m} / \mathrm{K}, c_{V, g}=3121 \mathrm{~J} / \mathrm{kg} / \mathrm{K}, c_{P, g}=5193 \mathrm{~J} / \mathrm{kg} / \mathrm{K}$ \\
Tank & $n=k=10, R=4.21 \mathrm{~m}, H=29.56 \mathrm{~m}^{2}, c_{w}=1 \times 5 \times 10^{2} \mathrm{~J} / \mathrm{K}, \rho_{w}=2700 \mathrm{~kg} / \mathrm{m}^{3}, d_{w}=0.1 \mathrm{~m}$, \\
& $S_{\text {valve }}=0.05 \mathrm{~cm}^{2}$ \\
\hline
\end{tabular}

\section{References}

[1] Osipov V. V., Daigle, M. J., Muratov, C. B., Foygel, M., Smelyanskiy, V. N., Watson, M. D., "Dynamical Model of Rocket Propellant Loading with Liquid Hydrogen", AIAA Journal of Spacecraft and Rockets, Vol. 20, No. 1, 2011, pp. 987-998.

[2] Daigle, M., Foygel, M., and V. Smelyanskiy, V., "Model-Based Diagnostics for Propellant Loading Systems", 2011 IEEE Aerospace Conference Proceedings, March 2011.

[3] Lin, W., Armfield, S. W., "Direct Simulation of Natural Convection Cooling in a Vertical Circular Cylinder", International Journal of Heat Mass Transfer, Vol. 42, 1999, pp. 4117-4130.

[4] Ho, S. H. and Rahman, M. M., "Transient Analysis of Cryogenic Liquid Hydrogen Storage Tank with Intermittent Forced Circulation", AIAA Journal of Thermophysics and Heat Transfer, Vol. 24, No. 2, 2010, pp. 374-380.

[5] Pu, W. L., Cheng, P., and Zhao, T. S., "An Experimental Study of Mixed Convection Heat Transfer in Vertical Packed Channels", AIAA Journal of Thermophysics and Heat Transfer, Vol. 13, No. 4, 1999, pp.517-521.

[6] Panzarella, C. H. and M. Kassemi, M., "On the Validity of Purely Thermodynamic Descriptions of Two-Phase Cryogenic Fluid Starage", Journal of Fluid Mechanics, 484, 2003, pp. 41-68. 
[7] Schallhorn, P., Campbell, D. M., Chase, S., Puquero, J., Fontenberry, C., Li, X., Grob, L., "Upper Stage Tank Thermodynamic Modelling Using SINDA/FLUINT”, AIAA-2006-5051, 44rd AIAA/ASME/SAE/ASEE Joint Propulsion Conference and Exhibit, Sacramento, CA, July 9-12, 2006.

[8] Wang, Y. Z., Hua, Y. X., and Meng, H., "Numerical Studies of Supercritical Turbulent Convective Heat Transfer of Cryogenic-Propellant Methane", AIAA Journal of Thermophysics and Heat Transfer, Vol. 24, No. 3, 2010, pp. 490-500.

[9] Barsi S., Moder, J., and Kassemi, M., "Numerical Investigation of LO2 and LCH4 Storage Tanks on the Lunar Surface”, AAIA 2008-4749, 44rd AIAA/ASME/SAE/ASEE Joint Propulsion Conference and Exhibit, Hartford, CT, July 21-23, 2008.

[10] Ahuja, V., Hosangadi, A., Mattick, S., and Lee, C. P., "Computational Analyses of Pressurization in Cryogenic Tanks", AIAA-2008-4752, 44rd AIAA/ASME/SAE/ASEE Joint Propulsion Conference and Exhibit, Hartford, CT, July 21-23, 2008.

[11] Khurana, T. K., Prasad, B. V. S. S. S., Ramamurthi, K., and Murthy, S. S., "Thermal stratification in ribbed liquid hydrogen storage tanks", International Journal of Hydrogen Energy, 31(15), pp. 2299-2309, December 2006.

[12] Moran, M. E., "Cryogenic Fluid Storage Technology Development: Recent and Planned Efforts at NASA”, Third Joint Army-Navy-NASA-Air Force (JANNAF) Spacecraft Propulsion Subcommittee (SPS) Meeting; 8-12 Dec. 2008; Orlando, Fl; United States.

[13] Doherty, M. P., Gaby, J. D., Salerno, L. J., and Sutherlin, S. G., "Cryogenic Fluid Management Technology for Moon and Mars Mission”, NASA/TM - 2010-216070, AAIA -2009-5632.

[14] Brennen, C. E., Fundamentals of Multiphase Flow, Cambridge University Press, 2005, p. 150.

[15] Schmidt, F. W., Henderson, R. E., and Wolgemuth, C. H., Introduction to Thermal Sciences, J. Wiley \& Sons, Second Ed., 1993, pp. 101, 179.

[16] Handbook of Single-Phase Convective Heat Transfer, ed by Kakac, S., Shah, R.K., and Aung W., John Wiley \& Sons, 1987, p. 12.1 .

[17] von Karman, T., 'On Laminar and Turbulent Friction”, National Advisory Committee for Aeronautics Technical Memorandum No. 1092, Washington D. C., September 1946.

[18] Eckert, E. R. G., and Jackson, T. W., "Analysis of Free-Convection Boundary Layer on Flat Plate”, National Advisory Committee for Aeronautics Technical Memorandum No. 2207, Washington D. C., September 1950.

[19] Yao, L. S., Molla, M., "Non-Newtonian Fluid Flow on a Flat Plate Part 1: Boundary Layer", AIAA Journal of Thermophysics and Heat Transfer, Vol. 22, No. 4, 2008, pp. 517-761.

[20] Yao, L. S., Molla, M., "Non-Newtonian Fluid Flow on a Flat Plate Part 2: Heat Transfer”, AIAA Journal of Thermophysics and Heat Transfer, Vol. 22, No. 4, 2008, pp. 762-765. 
[21] Schlichting H. and Gersten K., Boundary-Layer Theory, Springer, 8th Edition, Berlin, Heidelberg, New York, 2000 , p. 241.

[22] Landau, L. D. and Lifshitz, E. M., Fluid Mechanics, 2nd ed., Pergamon Press, 1987, p. 361. 